\title{
ON A VISUALIZATION OF THE CONVERGENCE OF THE BOUNDARY OF GENERALIZED MANDELBROT SET TO (n-1)-SPHERE
}

\author{
Andrzej Katunin, Kamil Fedio \\ Institute of Fundamentals of Machinery Design, Silesian University of Technology \\ Gliwice, Poland \\ andrzej.katunin@polsl.pl
}

\begin{abstract}
In this article we analyze the generalized Mandelbrot set in higher-order hypercomplex number spaces following both the Cayley-Dickson construction algebraic spaces and the spaces defined by Clifford algebras. The particular case of the generalized 3D quasi-Mandelbrot set was also considered. In particular, we investigated the increase of power of the iterated variable and proved that when this power tends to infinity, the Mandelbrot set is convergent to the unit circle. The same is true for the generalized Mandelbrot sets in higher-dimensional hypercomplex number spaces, i.e. when the power of iterated variable tends to infinity, the generalized Mandelbrot set is convergent to the unit $(n-1)$ sphere. The results of our investigation were visualized for the generalized Mandelbrot set in a complex number space and the generalized quasi-Mandelbrot set in a 3D Euclidean space.
\end{abstract}

Keywords: generalized Mandelbrot set, fractals, convergence, generalized sphere

\section{Introduction}

The Mandelbrot set (M-set) has been intensively studied by numerous researchers and research groups since it was discovered by Benoît Mandelbrot in 1979. This set was discovered based on earlier studies of Julia and Fatou in 1920 on dynamics of complex variable systems. Since the classic M-set was discovered, many generalizations have been developed to date. These generalizations include both modifications of initial analytic form describing the M-set (higher-order polynomial representation, generalized power value of an iterated variable) [1-3] and the generalizations to higher-dimensional multicomplex [4] and hypercomplex number spaces [5-8].

In the present study the authors considered both generalization types: generalization of power value of an iterated variable in M-set and generalization of M-set to the higher-order hypercomplex number spaces. The studies were focused on the specific conditions at which the boundary of M-set ceases to be fractal. These conditions can be disseminated to the generalized M-sets in higher-order hypercom- 
plex number spaces. Moreover, it was proven that the constant control parameter in the analytic equation of the generalized M-set is negligible when the power value of an iterated variable tends to infinity.

\section{Mandelbrot set and its generalizations}

The classic M-set can be presented in analytic form using the following recursive equation:

$$
z_{k+1}=z_{k}^{2}+c, z, c \in \mathbf{C},
$$

if $\left|z_{k}\right|_{\forall k \in \mathbf{N}}<2$ and the starting value of a sequence $\left\{z_{k}\right\}$ is usually set to zero, $c$ is the control parameter. The generalization of (1) with respect to the power value of an iterated variable $z$ was introduced in [9] and has the following form:

$$
z_{k+1}=z_{k}^{\alpha}+c, z, c \in \mathbf{C}, \alpha \in \mathbf{R} .
$$

In [9] the graphical analysis of the sets constructed this way with variable $\alpha$ and $c$ was performed. The authors of [9] stated that both variables have a significant influence on the shape of the M-set and determine its properties.

Further studies on M-sets (and J-sets as specific subproblems of the M-set) were performed for the generalizations of the M-set to the hypercomplex number spaces. Norton [5] and Griffin and Joshi [6] studied the classic M-set (1) generalized to the quaternionic and octonionic hypercomplex number spaces, respectively. In these cases the form of (1) remains unchanged, while $z, c \in \mathbf{H}$ for the quaternionic generalization and $z, c \in \mathbf{O}$ for the octonionic one. As it was stated in [7], further generalizations following the Cayley-Dickson construction are not possible since the generalized $\mathrm{M}$-sets are not closed under addition and multiplication anymore.

The generalizations of a generalized M-set (2) to higher-dimensional hypercomplex number spaces were introduced by Dixon et al. [10] and then investigated in more depth by Wang and Jin [8]. These generalizations were based on the Clifford algebras, which hold the condition of closing under addition and multiplication of M-set for an arbitrary-dimensional number space.

The last considered construction is a 3D quasi-M-set constructed by two enthusiasts: White and Nylander [11]. Since the canonical 3D M-set cannot exist due to the nonexistence of 3D analogue of a space of complex numbers or a space of quaternions, White and Nylander proposed the construction of an analogue of generalized 3D M-set (widely known as the Mandelbulb named after its characteristic shape) using spherical coordinates [12]:

$$
\langle x, y, z\rangle^{\alpha}=r^{\alpha}\langle\sin (\alpha \theta) \cos (\alpha \varphi), \sin (\alpha \theta) \sin (\alpha \varphi), \cos (\alpha \theta)\rangle,
$$


where $r=\sqrt{x^{2}+y^{2}+z^{2}}, \varphi=\arctan (y / x)$ and $\theta=\arctan \left(\sqrt{x^{2}+y^{2}} / z\right)$. Although this construction is not formally a 3D M-set, it exhibits the fractal nature of its surface and thus can be analyzed in terms of the investigated problem.

\section{Analysis of convergence}

Let us consider the generalized $\mathrm{M}$-set given by (2). The increase of $\alpha$ causes significant changes in the shape of the M-set, when $\alpha$ is considerably small. However, when the value of $\alpha$ increases the shape of the M-set it tends to the circular shape. This leads to the formulation of a following theorem.

Theorem. The boundary of a generalized M-set of type (2) tends to unit circle when $\alpha \rightarrow \infty$.

Proof. Suppose $c=0$ in (1), which simplifies (1) to the form:

$$
f: z_{k+1}=z_{k}^{2}, z \in \mathbf{C} .
$$

In case of application of the recursive procedure following (4) one obtains three possibilities: when $\left|z_{k}\right|>1$ the generated point is the repelling point, i.e. $\left|z_{k}\right|$ will grow successively for $k \rightarrow \infty$; when $\left|z_{k}\right|<1$ the generated point is the attractive point, i.e. $\left|z_{k}\right| \rightarrow 0$ when $k \rightarrow \infty$; and finally, when $\left|z_{k}\right|=1$ one obtains the point which constitutes the boundary of the generated set. Accordingly, there exist two attracting points $x_{0}$ and $x_{\infty}$ of $f$ at 0 and $\infty$ for $|f(x)|=x$ and $0 \leq\left|f^{\prime}(x)\right|<1$, and a dense set of repelling points $x_{k}$ of $f$ for the cases $\left|f^{\prime}(x)\right|<-1$ or $\left|f^{\prime}(x)\right|>1$. Following this, during the iteration procedure of $f(x)$ for $x_{\omega}=\exp (i \omega)$, one obtains the unit circle constructed from vectors $\vec{v}=\left[x_{0}, x_{\omega}\right]$ with a radius defined by $\|\vec{v}\|$ in a complex number space since the iteration corresponds to the rotation by $\omega$.

Now, suppose $\alpha \rightarrow \infty$ in (2). In this case during the iteration procedure one obtains the same possibilities of $z_{k}$ as for (4). The M-set tends to the unit circle for arbitrary value of $c$. This causes that in the limit case (when $\alpha \rightarrow \infty$ ) the resulted set has a non-fractal boundary.

Corollary 1. The proof of the above-presented theorem can be generalized to the generalized $\mathrm{M}$-sets in the considered hypercomplex number spaces, namely:

$$
\begin{aligned}
& z_{k+1}=z_{k}^{\alpha}+c, z, c \in \mathbf{H}, \alpha \in(-\infty,-2] \cup[2, \infty), \\
& z_{k+1}=z_{k}^{\alpha}+c, z, c \in \mathbf{O}, \alpha \in(-\infty,-2] \cup[2, \infty),
\end{aligned}
$$




$$
z_{k+1}=z_{k}^{\alpha}+c, z, c \in C l(\cdot, \cdot), \alpha \in(-\infty,-2] \cup[2, \infty),
$$

where $\mathbf{H}, \mathbf{O}$ and $C l(\cdot$,$) denotes a quaternion, octonion and Clifford number spaces,$ respectively, since if $\alpha \rightarrow \infty$, then $z_{k}^{\alpha} \rightarrow \infty$ and $c$ has no influence on the resulted set. Depending on the considered hypercomplex number space, the resulted set will be the unit $(n-1)$-sphere, where $n$ is the dimension of a considered space.

Proof. Similarly as previously one can recall three mentioned possibilities for the values of $\left|z_{k}\right|$. Considering (4) and replacing $z \in \mathbf{C}$ by $z \in \mathbf{H}, z \in \mathbf{O}$ and $z \in C l(.$,$) ,$ subsequently, one can define the attractive points $x_{0}$ and $x_{\infty}$ of $f$ at 0 and $\infty$ that satisfy $|f(x)|=x$ and $0 \leq\left|f^{\prime}(x)\right|<1$, and a dense set of repelling points $x_{k}$ of $f$ for the cases $\left|f^{\prime}(x)\right|<-1$ or $\left|f^{\prime}(x)\right|>1$ for each of the mentioned number spaces. Considering this definition $f(z)=z^{\alpha}$ and $f^{m}(z)=z^{\alpha^{m}}$ it can be observed that when $m \rightarrow \infty$, if $\left|z_{k}\right|>1$ then $f^{m}(z) \rightarrow \infty$, if $\left|z_{k}\right|<1$ then $f^{m}(z) \rightarrow 0$ and if $\left|z_{k}\right|=1$ then $f^{m}(z)$ is a boundary of a unit $(n-1)$-sphere. Similar conclusions can be found for the generalized Julia set [13].

Corollary 2. The proof of the above-presented theorem can be generalized also to the $3 \mathrm{D}$ quasi-M-set. In the case when $\alpha \rightarrow \infty$, the resulted quasi-M-set tends to the unit 2-sphere.

Proof. Since the form of (3) differs from previously investigated recursive equations one can use the trigonometric approach. In this case the iterative procedure can be presented as

$$
f: B(z)=z^{\alpha}+c, z, c \in \mathbf{R}^{3}, \alpha \in(-\infty,-2] \cup[2, \infty),
$$

where $B$ is a generalized $3 \mathrm{D}$ quasi-M-set and $z$ is given by a triplet $\left(r^{\alpha}, \alpha \varphi, \alpha \theta\right)$, which is a point in spherical coordinates. Following this, one can deduce that for $\alpha \rightarrow \infty$ equation (8) reduces to $f: B(z)=z^{\alpha}, f(z)=z^{\alpha}$, and the only parameter of an above triplet that has an influence on the distance of a generated point from the origin is $r$. Obviously, when $\alpha \rightarrow \infty$, if $r>1$ then $f(z) \rightarrow \infty$, if $r<1$ then $f(z) \rightarrow 0$ and if $r=1$ then $f(z)$ forms a boundary of a unit 2-sphere.

In order to analyze the phenomenon, several computer simulations for the generalized $\mathrm{M}$-set in a complex number space and the generalized quasi-M-set in 3D Euclidean space were performed. In the first case a Matlab ${ }^{\circledR}$ routine was used for implementation of the recursive procedure of the M-set known as the Escape Time Algorithm (see e.g. [14]). The generation of subsequent points was based on a loop with a predefined number of iterations (assumed as 100), where the initial value $z_{0}$ 
was defined by a grid with a resolution of resulted image. The loop iteration was performed on (2) and additionally contained a condition: $\left|z_{k}\right| \leq 2$, where the value 2 is the Bailout value, for determination of interior and exterior parts limited by the $\mathrm{M}$-set and definition of the coloring properties, i.e. the interior part (inside the M-set) was colored black, while the colors defined for the exterior part (outside the M-set) denote the speed of convergence to infinity. The evolution of the shape of M-sets for variable $\alpha$ with a constant $c$ was presented in Figure 1.
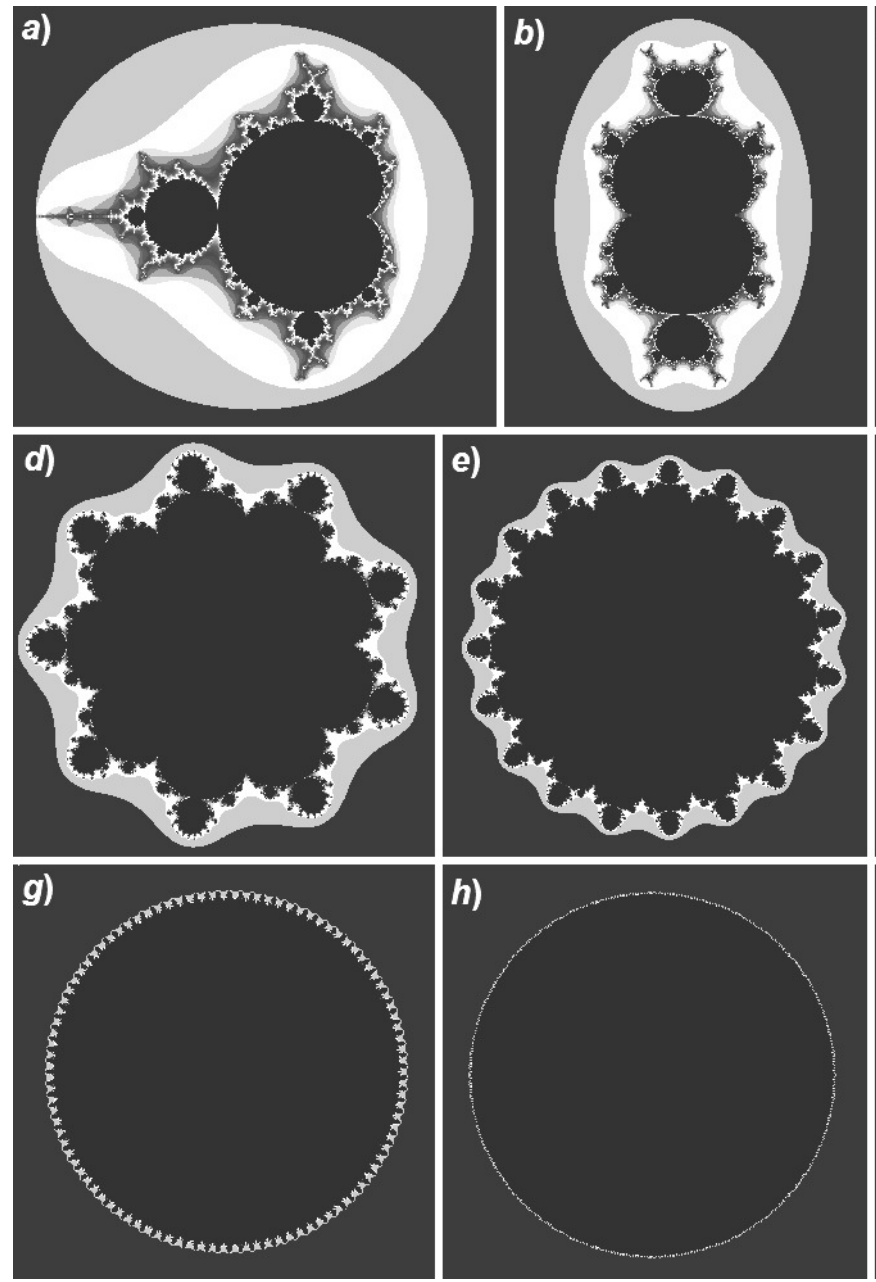

Fig. 1. M-sets for: a) $\alpha=2$, b) $\alpha=3$, c) $\alpha=5$, d) $\alpha=10$, e) $\alpha=20$, f) $\alpha=50$, g) $\alpha=100$, h) $\alpha=500$, i) $\alpha=100000$ in a complex number space

The 3D quasi-M-set was visualized using Chaos Pro software, where the algorithm proposed by Nylander and White was implemented. In this case the recursion 
is performed following equation (3) using the modified Escape Time algorithm with input parameters of Bailout value and a power $\alpha$ of a quasi-M-set. The obtained boundary is then rendered using available color maps.

a)
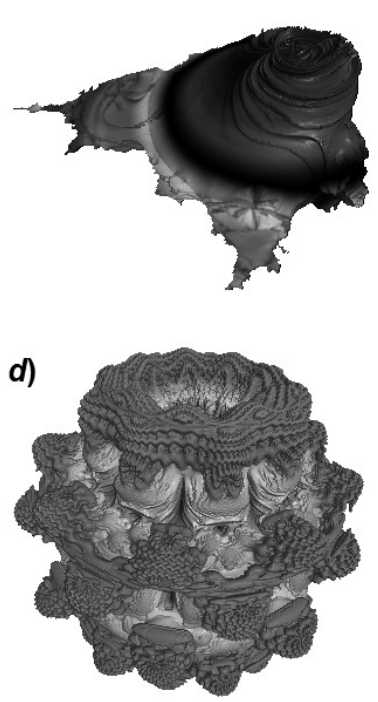

g)

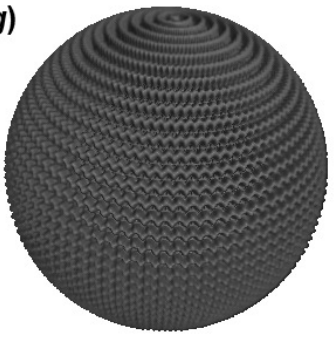

b)

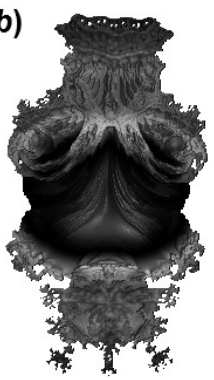

e)

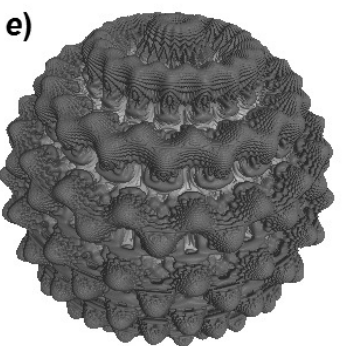

h)

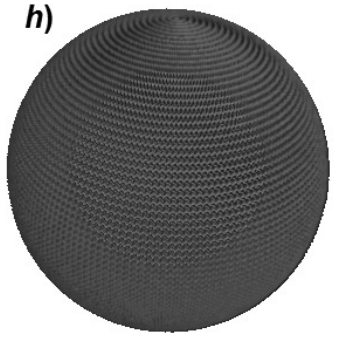

c)

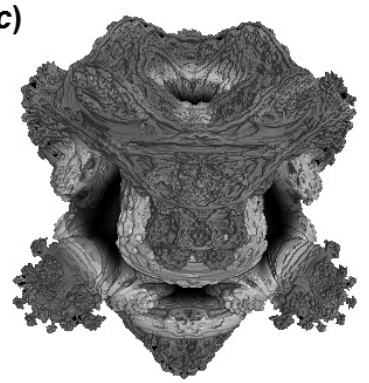

f)

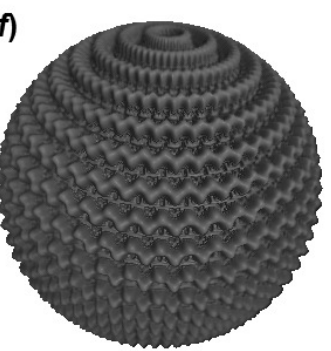

i)

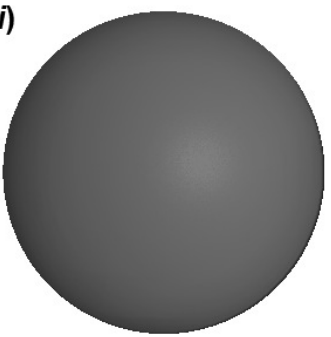

Fig. 2. Quasi-M-sets for: a) $\alpha=2$, b) $\alpha=3$, c) $\alpha=5$, d) $\alpha=10$, e) $\alpha=20$, f) $\alpha=50$, g) $\alpha=100$, h) $\alpha=200$, i) $\alpha=5000$ in a 3D Euclidean space

One can observe the convergence of M-set to the unit circle for $\alpha=100$ (Fig. 1g) and for $\alpha=100000$ (Fig.1i) the unit circle is observed for the assumed resolution of a rendered picture. Based on the analysis of visual representation of the M-sets for various $\alpha$ it is obvious that when $\alpha \rightarrow \infty$ the influence of $c$ becomes negligible. Similarly, the evolution of 3D quasi-M-sets for variable $\alpha$ with a constant $c$ was presented in Figure 2. In the case of these sets the convergence to the unit sphere can be observed. Similarly to the M-sets in a complex number space the 3D quasi-M-set with $\alpha=5000$ (Fig. 2i) seems to be a sphere considering the assumed resolution of a rendered picture. 


\section{Conclusions}

The performed analysis of the generalized M-set to the higher-order hypercomplex number spaces, when a power of iterated variable in the recursive equation of M-set tends to infinity, shows that the analyzed set is convergent to (n-1)-sphere, where $n$ denotes the dimension of a considered space. The visualization of evolution of the generalized M-set in a complex number space, as well as the generalized quasi-M-set in 3D Euclidean space with increasing power of iterated variable additionally confirmed the formulated theorem. It was also shown that when the power of iterated variable tends to infinity, the influence of a constant control parameter is negligible.

\section{References}

[1] Gujar U.G., Bhavsar V.C., Fractals from $z \leftarrow z^{\alpha}+c$ in the complex $c$-plane, Comput. Graph. 1991, 15, 441-449.

[2] Gujar U.G., Bhavsar V.C., Fractals images from $z \leftarrow z^{\alpha}+c$ in the complex $z$-plane, Comput. Graph. 1992, 16, 45-49.

[3] Wang X.Y., Liu X.D., Zhu W.Y., Gu S.S., Analysis of $c$-plane fractal images from $z \leftarrow z^{\alpha}+c$ for $(\alpha<0)$, Fractals 2000, 8, 307-314.

[4] Garant-Pelletier V., Rochon D., On a generalized Fatou-Julia theorem in multicomplex spaces, Fractals 2009, 17, 241-255.

[5] Norton A., Julia sets in quaternions, Comput. Graph. 1989, 2, 267-278.

[6] Griffin C.J., Joshi G.C., Octonionic Julia sets, Chaos Soliton. Fract. 1992, 2, 11-24.

[7] Katunin A., On the symmetry of bioctonionic Julia sets, J. Appl. Math. Comput. Mech. 2013, 12, 23-28.

[8] Wang X.Y., Jin T., Hyperdimensional generalized M-J sets in hypercomplex number space, Nonlinear Dyn. 2013, 73, 843-852.

[9] Lakhtakia A., Varadan V.V., Messier R., Varadan V.K., On the symmetries of the Julia sets for the process $z \rightarrow z^{p}+c$, J. Phys. A: Math. Gen. 1987, 20, 3533-3535.

[10] Dixon S.L., Steele K.L., Burton R.P., Generation and graphical analysis of Mandelbrot and Julia sets in more than four dimensions, Comput. Graph. 1996, 20, 451-456.

[11] http://www.skytopia.com/project/fractal/mandelbulb.html (accessed on Dec 16, 2014).

[12] http://www.bugman123.com/Hypercomplex/index.html (accessed on Dec 16, 2014).

[13] Blancharel P., Complex analytic dynamics on the Riemann sphere, Bull. Am. Math. Soc. 1984, $11,88-144$.

[14] Liu X., Zhu Z., Wang G., Zhu W., Composed accelerated escape time algorithm to construct the general Mandelbrot set, Fractals 2001, 9, 149-153. 